\title{
Development of a Virtual Electric Wheelchair - Simulation and Assessment of Physical Fidelity Using the Unreal Engine 3
}

\author{
Marc Herrlich ${ }^{1}$, Ronald Meyer ${ }^{1}$, Rainer Malaka ${ }^{1}$, and Helmut Heck ${ }^{2}$ \\ ${ }^{1}$ Digital Media Group \\ TZI, University of Bremen \\ ${ }^{2}$ Research Institute Technology and Disability (FTB) \\ of Evangelische Stiftung Volmarstein
}

\begin{abstract}
This paper demonstrates how an existing game technology as a component off-the-shelf can be used as a basis to build a serious game for assistive technology for disabled people.Using the example of a virtual electric wheelchair simulator, we present how to use a computer game physics engine to achieve a realistic simulation of driving an electric wheelchair in a virtual environment. Focus of the paper is the conversion of driving characteristics of prevalently used electric wheelchairs into the virtual physics system of the used computer game engine. The used parameters are systematically balanced between the virtual and the real world to evaluate the realism of the driving characteristics of an electric wheelchair using the integrated physics simulation of the Unreal Engine 3.
\end{abstract}

Keywords: serious games, virtual electric wheelchair, COTS, physics simulation.

\section{Introduction}

Users of wheelchairs quite often face limitations and barriers when moving in public and private environments due to the fact that many environments have not been designed to be accessible for wheelchairs [8. Awareness and knowledge of the needs of disabled people needs to be increased, especially for people who provide public services and for people who design public environments. A serious game [6] can be a good tool to rise awareness. For having a connection between the physical and the virtual world a certain degree of realism in the virtual world is necessary, meaning in our case the physical driving behavior of the wheelchair should be approximated as good as possible. The Unreal Engine 3 (UE3), a state of the art game engine, includes the Nvidia PhysX physics engine, and thereby offers the functionality to realize a virtual driving simulator for a wheelchair.

The quality of the approach to simulate the specific driving characteristics is evaluated in this paper including the application of the working wheelchair model in a virtual environment. The outcome of this research is a tool that gives a virtual experience of typical situations of wheelchair users, and can also be used by people who have to design accessible environments. 


\section{A Virtual Wheelchair Simulator as a Serious Game}

The application of simulation and virtual reality technologies can today be assessed as essential and cost-effective [5] 10, 11. The term "Serious Games" specifies games that combine simulation and virtual reality with game play to train people in decision-making, problem-solving, and for other educational purposes in a "playful" way. The application of serious games has its origins mainly in the military 9] and the industrial sector. But also the medical and rehabilitation sector's attention is growing for the application of serious games. For instance, Jack et al. 4 are using virtual reality to help stroke patients in their recovery. The use of game engines as components off-the-shelf is a popular way to lower costs for developing virtual reality simulations or serious games [9].

The virtual wheelchair simulator described in this paper has been developed in cooperation with the Research Institute Technology and Disability [3] (FTB).During the development phase, an idea exchange took place between the two collaborating institutes, FTB and Digital Media Group at Bremen. Necessary data for implementation was either available at the FTB or could be "experienced" and researched in their laboratories. This included for example trying out different models of existing and commonly used electric wheelchairs. This close collaboration allowed the use of realistic data and the focus on requirements and needs of wheelchair users. FTB was furthermore capable of providing specification sheets of state-of-the-art electric wheelchairs that were essential for the implementation of a realistic driving behavior of the virtual wheelchair.

\section{Driving Characteristics of a Wheelchair}

The driving characteristics of an electric wheelchairare based on its respective purpose and application area. Wheelchairs that are predominantly used outside are preferably equipped with a direct steering technology and are designed to be driven at higher speeds without sliding in curve maneuvers. Wheelchairs primarily used for indoor driving purposes are usually equipped with an indirect steering technology and lower maximum speeds are required or even imposed to allow navigation in narrow and tight spaces. As a wheelchair driver usually needs to be able to use his wheelchair, both, inside and outside there are also hybrid models of wheelchairs that try to find a good compromise between the different requirements of indoor and outdoor wheelchairs.

Besides speed and maneuverability, wheelchairs have to fulfill safety characteristics like its minimum turning radius, an ability to get over barricades and obstacles, and tilt resistance in slopes and cross slopes. These characteristics are highly dependent on the construction and steering systems used in the different wheelchair models. For the research that we describe in this paper, the wheelchairs of the manufacturer Meyra have been used as a template. An overview about their specifications is given in table 1. 
Table 1. Wheelchair models and their specifications engineered by Meyra

\begin{tabular}{|l|c|c|c|c|c|c|}
\hline model & steering type & turn radius & max barricade & max slope & max cross slopes & tilt resistance \\
\hline Clou & indirect & $1 \mathrm{~m}$ max. & $60 \mathrm{~mm}$ & $12 \%$ & $12 \%$ & $15 \%$ \\
\hline Compact 905 & indirect & $0,83 \mathrm{~m}$ max. & $60 \mathrm{~mm}$ & $15 \%$ & $15 \%$ & $15 \%$ \\
\hline Allround 903 & indirect & $0,85 \mathrm{~m}$ max. & $80 \mathrm{~mm}$ & $15 \%$ & $15 \%$ & $15 \%$ \\
\hline Allround 900 C & indirect & $0,88 \mathrm{~m}$ max. & $75 \mathrm{~mm}$ & $15 \%$ & $15 \%$ & $15 \%$ \\
\hline Optimus 2 & direct & $1,2 \mathrm{~m}$ & $110 \mathrm{~mm}$ & $18 \%$ & $18 \%$ & $28 \%$ \\
\hline Touring 928 & direct & $1,2 \mathrm{~m}$ & $110 \mathrm{~mm}$ & $18 \%$ & $18 \%$ & $28 \%$ \\
\hline
\end{tabular}

\section{Unit Conversion and Virtual Driving Characteristics}

The simulation and transfer of a process from the physical world to the virtual world of UE3 requires to abstract all necessary data. The most basic unit that had to be conversed was a length unit for representing space in virtuality. Since the development of the first Unreal game and its release in 1998 the developers have been using the term "Unreal Unit" to refer to an elementary spatial unit. This specification has not changed since finishing the development of the game Unreal in 1998 and is still being used for determination of length etc. The application of Spring and Jennings virtualization taxonomy [7] led to the conversion of the length units as illustrated in table 2 .

Table 2. Comparison: real world unit - virtual Unreal Unit

\begin{tabular}{|l|l|}
\hline reality & \multicolumn{1}{|c|}{ virtuality } \\
\hline $487.68 \mathrm{~cm}$ & $256 \mathrm{UU}$ \\
\hline 1 meter & $52.5 \mathrm{UU}$ \\
\hline $1 \mathrm{foot}$ & $16 \mathrm{UU}$ \\
\hline $1 \mathrm{~cm}$ & $0.525 \mathrm{UU}$ \\
\hline 0.75 inches & $1 \mathrm{UU}$ \\
\hline
\end{tabular}

The current version of the wheelchair simulator allows steering the virtual wheelchair by keyboard and mouse or a gamepad. UE3 provides any kind of data input as the script engine includes classes that can handle input data from any interface. The use of any input device is possible that way, preferably a steering device should be chosen that is relatively similar to the "joystick"-like control devices used by real electric wheelchairs.

\section{Assembling the Virtual Reality Wheelchair}

UE3 allows a versatile use of self-assembled assets to augment the game or to create a game modification from scratch. The $3 \mathrm{~d}$ model of the virtual wheelchair was created using Autodesk's 3d modeling software "3D Studio Max" and imported to the engine using a converter provided by Epic Games. To allow the 
wheelchair to be used in multiple scenario types, the base model of a hybrid usable wheelchair fits best to meet the requirements of diverse scenarios for a Serious Game. Therefore, the model of an indirectly steering wheelchair with rear-mounted drive has been chosen for conversion.

The selection of the camera perspective plays an important role for the player's immersion in the simulation. The used camera has its origin located according to the wheelchair driver's head position. This results in a first person view, which provides a stronger feeling of immersion to the player.

\section{Scenario Development}

The employment of the virtual wheelchair is predetermined for hybrid use. This allows to choose a scenario from an everyday situation in the life of a wheelchair driver: the access of a supermarket. Supermarkets should be accessible also for people with reduced mobility, e.g. users of rollators or wheelchairs. To guarantee a barrier-free access to such a public building the German Institute for Standardization (DIN) has approved several standards for accessing public buildings [2] and places [1]. The standardized values provided by the DIN have been abstracted to the engine by using the previously developed conversion units.

For constructing the virtual scenario, the level editor accompanying UE3, called "UnrealEd" was used. The supermarket has been created on an elevated stage to include the use of a wheelchair ramp. The scenario provides several challenges for a wheelchair driver. Narrow approaches like doors and close racks (cf. figure 1) demonstrate the difficulty that most wheelchair drivers have in everyday life, even accessing a place that is constructed with respect to established standards.

UE3 features numerous options in recording actions and events that occur in the engine. Several of these values were used to either adapt the physical behavior (driving characteristics) of the wheelchair or to detect important events like

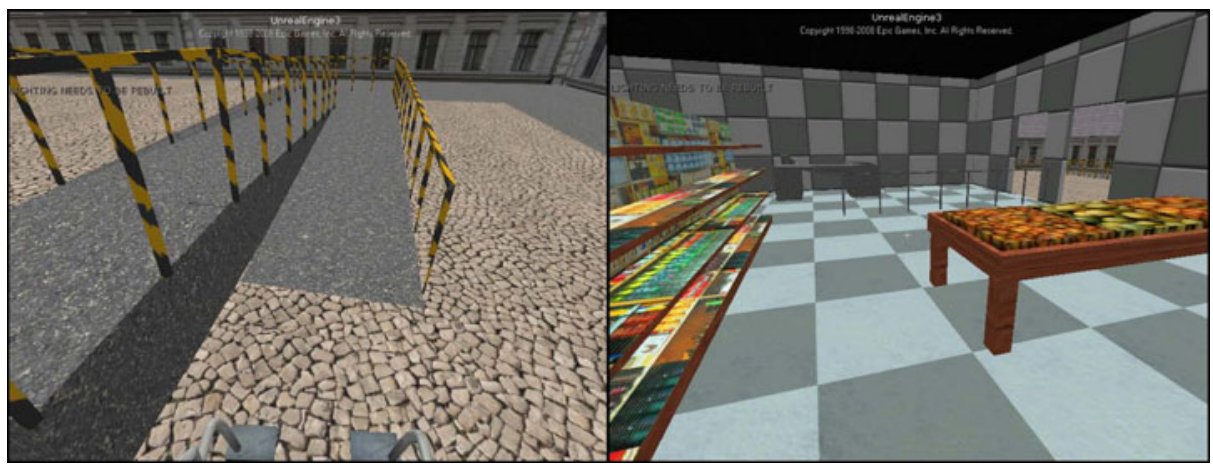

Fig. 1. Left: Accessing a wheelchair ramp with the VR-wheelchair; Right: Obstacles and narrow approaches inside the VR supermarket 
collisions between the wheelchair and objects in the game world. The detected values can be used to trigger following events or simply be passed to the game console for monitoring. The use of recording and triggering events provides several options to add game play to the simulation and to make it a Serious Game in the end. A basic idea of the game play was the simple marking of the route that has to be accomplished by the virtual wheelchair driver in order to have a challenge. This route is marked with a row of red light spots (cf. figure 2), which turn green when they are approached correctly.

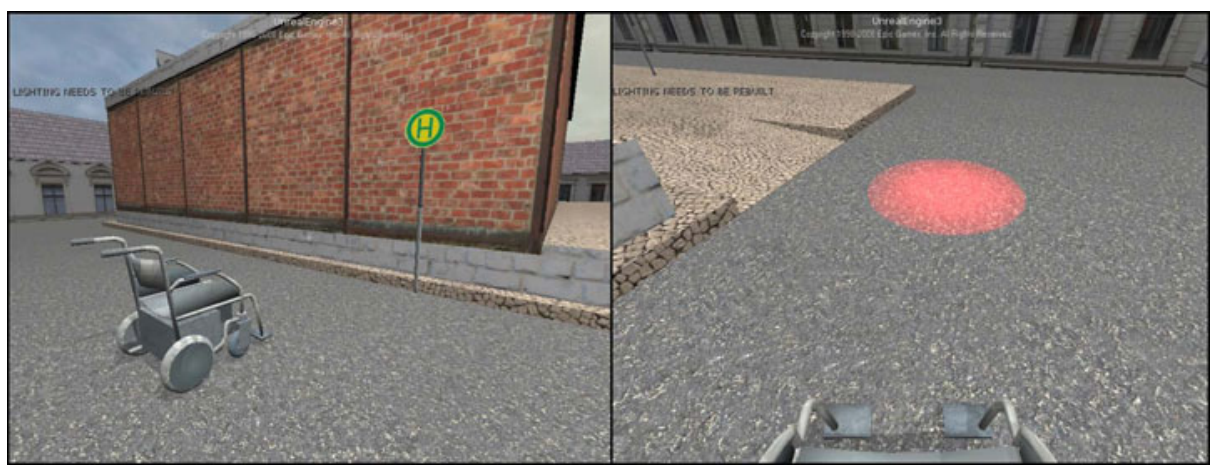

Fig. 2. Left: VR wheelchair in 3rd person view at starting point; Right: Trigger spot that turns green when correctly approached

\section{Adaptation and Testing of Driving Characteristics}

To achieve an as realistic as possible driving behavior of the virtual wheelchair, the driving characteristics and their appropriate conversion to the virtual domain were experimentally explored by systematically trying different parameter combinations and checking results for certain metrics and measurements related to the physical world, like turning radii. A highly experimental and empirical approach like this was required because no specifications of comparable values for the driving parameters of the virtual vehicle models of UE3 exists. For the adaptation of each driving characteristic from the physical world to the virtual world of the Unreal Engine a set of specific tests had to be developed. The guideline values for conversion were taken from real electric wheelchairs and are listed in table 1 in section 3 .

\subsection{Speed}

Vehicles assembled in UE3 are provided with a parameter for speed. But as there is no factor yet for the conversion of a speed unit from a real world unit like kilometers per hour to the virtual world of UE3, a factor had to be determined experimentally. As time and length are the basis for this factor, a scenario was created, which was used to simply measure speed. For this, a game world was 
created in the UE3 level editor including two trigger points placed at the exact distance of 5250 Unreal Units, which is correspondent to a hundred meters in the physical world (cf. table 2). These two triggers write a time value to the console each time they detect a collision with the virtual wheelchair. The length of the time interval and the predetermined length of a 100 meters can now be used to calculate the conversion factor for speed from the physical to the virtual world by knowing the vehicles virtual speed parameter value. The measurements and calculations resulted in 14.50 Unreal Speed Units equal one kilometer per hour in the physical world. A speed of six kilometers per hour was determined to be applied to the virtual wheelchair because this is a typical maximum speed of electric wheelchair models.

\subsection{Braking and Acceleration}

Braking and acceleration are important factors to define a reliable handling and a wheelchair's safety. A vehicle in UE3 can be provided with several acceleration factors that are dependent on the current speed of the vehicle. This factor is used to adjust the acceleration behavior of the virtual wheelchair to that of its real equivalent. Smooth and controlled acceleration guarantees safe approaching to barricades for example. Even more important are braking maneuvers: the intention of the wheelchair driver to come to a full stop, i.e. maximum braking or "de-acceleration", must not be that strong to toss the driver from the wheelchair seat, but the stopping distance must be as short as possible. The engine provides several torque and brake factors for vehicles that can be adjusted to behave like the braking of a real wheelchair.

\subsection{Slopes and Cross Slopes}

The safety approved values for a tilt of wheelchair models are between twelve and $18 \%$ pitch (cf. table1). The absolute maximum average value for the tilting of a wheelchair is at $28 \%$. The maximum value is higher due to a safety buffer before the wheelchair actually tilts over and endangers or even harms the driver. A realistic tilt value is desirable to make the simulation as realistic as possible. To adapt the tilting value to the virtual wheelchair model, the center of mass (COM) of the object was manipulated. UE3 allows one to move the center of mass in XYZ space. A movement of the COM in $+\mathrm{Z}$ or $-\mathrm{Z}$ direction which accords to "up" or "down" directions, leads to a change of the tilt value. To test the tilting behavior a row of ramps with increasingly raised pitches was created in the UE3 level editor. With these the tilting behavior could be adapted to the predefined values. Cross slopes are handled in the same way as values and parameters are the same except the wheelchair is standing transverse to the slope.

\subsection{Cornering}

A cornering can hold a dangerous situation for a wheelchair driver, especially when driving at higher speed rates. An electric wheelchair's steering behavior is 
usually linked to its speed. The safe turning radius dilates with the increase of speed. This behavior could be directly transfered to UE3 by having a parameter that allows the steering value to be adapted to the current vehicle speed.

\subsection{Barriers and Maneuverability}

Barriers and obstacles can be of a different manner. A curbstone for example can be surmounted with a wheelchair depending on its front wheel size. UE3 allows to adapt the height of all assembled wheels on the vehicle. This allows principally an adaptation of the model to any desired wheel height to make the virtual wheelchair surmount barriers that conform to standardized values. A cornering in a narrow corridor can also be considered as an obstacle. A wheelchair's turning radius significantly determines its maneuverability. The maneuverability of the virtual wheelchair has been tested by constructing circular platforms with different radii. Each wheel of the virtual wheelchair was monitored due to whether it is touching the ground or not. The test was invalid as soon as one wheel was off ground. A minimum turn radius of approximately two meters or 105 Unreal Units was ascertained for the virtual wheelchair. This big turn radius is due to the turning fulcrum lying on one of the rear wheels when turning. The overall width of the virtual wheelchair is 50.7 Unreal Units or 96 centimeters.

\section{Evaluation of Driving Characteristics}

The virtual wheelchair was assembled in UE3 by using a basis model that allows the creation of wheeled vehicles. The virtual wheelchair model was conceptualized as an indirectly steered wheelchair with rear mounted gear. Unfortunately, the indirect steering method could not be transfered directly to the engine. The physics engine did not allow indirect forces on the front wheels, which resulted in unexpected behavior in the simulation. A real indirect steering technology usually allows an individual torque control of each rear wheel and no torque on the front wheels. The different torque values are used for steering, thus the turning fulcrum is located in the middle between the rear wheels. This also affects the cornering radius significantly. With a correctly working physics model the cornering radius would be halved to approximately 52.5 Unreal Units or one physical meter (cf. section 7.5). The front wheels should also be able to turn freely through 360 degrees following the forces of the rear wheels. The conversion of a directly steered wheelchair model is the better choice when using a wheeled vehicle physics model from UE3 as the implemented model follows the behavior of a directly steered wheelchair.

\section{Conclusion}

The results of this research show how physics simulation in a game engine is used to create a virtual electric wheelchair simulation whose physical behavior is 
adequate to create realistic scenarios for everyday situations from the real life of wheelchair drivers. Engine specific tests were run to adapt the driving behavior to the one of a real wheelchair. As the used vehicle model of the engine did not fulfill all requirements to the chosen wheelchair model either another wheelchair model can be implemented or a different vehicle model in UE3 should be used in future work. Methods were applied that allow a correlated conversion of real units into the virtual system. We plan to employ our wheelchair simulator as a support tool in education of rehabilitation personnel and to use it as the basis for building serious games, probably in the form of an Unreal 3 "modification", around it to raise awareness for the difficulties of electric wheelchair users in everyday life.

\section{References}

1. DIN 18 024-1, 1998-01 Strassen, Plätze, Wege, öffentliche Verkehrs- und Grünanlagen sowie Spielplätze (1998), http://nullbarriere.de/din18024-1.htm (accessed on March 10, 2010)

2. DIN 18 024-2, 1996-11 Öffentlich zugängige Gebäude und Arbeitsstätten, http://nullbarriere.de/din18024-2.htm (accessed on March 10, 2010)

3. Forschungsinstitut Technologie und Behinderung. Website, http://www.ftb-net.com (accessed on March 10, 2010)

4. Jack, D., Boian, R., Merians, A., Tremaine, M., Burdea, G., Adamovich, S., Recce, M., Poizner, H.: Virtual reality-enhanced stroke rehabilitation. IEEE Transactions on Neural Systems and Rehabilitation Engineering 9(3), 308-318 (2001)

5. Niniss, H., Nadif, A.: Simulation of the behaviour of a powered wheelchair using virtual reality. In: 3rd International Conference on Disability, Virtual Reality and Associated Technologies 2000, pp. 9-14 (2000) (citeseer)

6. Sawyer, B., Smith, P.: Serious games taxonomy. In: Slides from the Serious Games Summit at the Game Developers Conference (2008)

7. Spring, M., Jennings, M.: Virtual reality and abstract data: Virtualizing information. Virtual Reality World 1(1) (1993)

8. Stredney, D., Carlson, W., Swan II, J., Blostein, B.: The determination of environmental accessibility and ADA compliance through virtual wheelchair simulation. Presence-Teleoperators and Virtual Environments 4(3), 297-305 (1995)

9. Susi, T., Johannesson, M., Backlund, P.: Serious games-An overview. Skövde: University of Skövde (Technical Report HS-IKI-TR-07-001) (2007)

10. Virvou, M., Manos, K., Katsionis, G.: Virtual Reality edutainment: cost-effective development of personalised software applications. In: Gavrilova, M.L., Gervasi, O., Kumar, V., Tan, C.J.K., Taniar, D., Laganá, A., Mun, Y., Choo, H. (eds.) ICCSA 2006. LNCS, vol. 3980, p. 251. Springer, Heidelberg (2006)

11. Voas, J.: Cots software: the economical choice? IEEE Software 15(2), 16-19 (1998) 